\title{
Numerical simulation of drug release from collagen matrices by enzymatic degradation
}

\author{
F. A. Radu - M. Bause - P. Knabner - W. Friess • \\ I. Metzmacher
}

Received: 20 January 2007 / Accepted: 15 June 2007 / Published online: 24 July 2008

(C) The Author(s) 2008

\begin{abstract}
Biodegradable collagen matrices have become a promising alternative to synthetic polymers as drug delivery systems for sustained release. Previously, a mathematical model describing water penetration, matrix swelling and drug release by diffusion from dense collagen matrices was introduced and tested (cf. Radu et al. in J. Pharm. Sci. 91:964-972, 2002). However, enzymatic matrix degradation influences the drug release as well. Based on experimental studies (cf. Metzmacher in Enzymatic degradation and drug release behavior of dense collagen implants. Ph.D. thesis, LMU University of Munich, 2005), a mathematical model is presented here that describes drug release by collagenolytic matrix degradation. Existence and uniqueness of a solution of the model equations is reviewed. A mixed Raviart-Thomas finite element discretization for solving the coupled system of partial and ordinary differential equations is proposed and
\end{abstract}

Communicated by G. Wittum.

This research was supported by the Deutsche Forschungsgemeinschaft (DFG, Germany) Grant FR 1089/4-1.

\section{F. A. Radu ( $\varangle)$}

Max-Planck-Institut für Mathematik in den Naturwissenschaften, Inselstr. 22, 04103 Leipzig, Germany

e-mail: florin.radu@ufz.de

Present address:

F. A. Radu

Helmholtz Center for Environmental Research, UFZ,

Permoserstr. 15, 04318 Leipzig, Germany

M. Bause · P. Knabner

Department Mathematik, Universität Erlangen-Nürnberg,

Martensstr. 3, 91058 Erlangen, Germany

W. Friess · I. Metzmacher

Department für Pharmazie Lehrstuhl für Pharmazeutische

Technologie und Biopharmazie, Ludwig-Maximilians Universität

München, Butenandtstr. 5, 81377 München, Germany analyzed theoretically. The model is verified by a comparison of numerically calculated and experimentally measured data and, in particular, investigated by a parameter sensitivity study. For illustration, some concentration profiles of a twodimensional simulation are shown.

\section{Introduction}

Collagen is the major constituent of connective tissue; cf. [8]. Due to its wide distribution in the mammalian body, it has become a promising material for biodegradable drug delivery systems. Dense collagen matrices for sustained release of higher weight drugs such as proteins or polysaccharides offer an alternative to implants based on synthetic polymers; cf. [8]. Especially drug release from insoluble collagen devices is of interest since drug liberation can be controlled by swelling and enzymatic degradation. Initially the matrix swells and drug is released by diffusion. This phenomenon was described previously in a mathematical model; cf. [18]. Subsequently, degradation by collagenolytic enzymes occurs and the release rate becomes additionally dependent on the enzymatic binding and cleavage rate.

For designing and optimizing such biodegradable drug delivery systems there is a strong need of mathematical models that describe both, matrix degradation and drug release, by considering all the relevant processes occuring. In a recent work of the authors, the enzymatically catalyzed matrix degradation and simultaneous drug delivery was studied experimentally by measurements; cf. $[15,16]$. Based on these experimental investigations, a mathematical model was formulated and validated by a comparison of measurements and numerical calculations. Other models that were published in the literature to describe collagen matrix degradation and drug release either oversimplify (cf. [12,22]) or are less 
complete than the model that is given in Sect. 2. In [24], Tzafriri et al. suggest a model for enzymatic matrix degradation that is similar in complexity with the one given here, but the authors describe the matrix degradation differently and, moreover, do not consider the drug release.

Whereas in [16] we focused on experimental investigations of the material and the identification of all relevant processes, in this work the mathematical model and numerical techniques for solving the equations are considered more in detail. In particular, for the mathematical model the existence and uniqueness of a solution is reviewed and an error estimate is proved for a Raviart-Thomas mixed finite element discretization scheme that is applied here. The latter is done for a semilinear model problem that is of the same mathematical structure as the governing equations of the overall system. The innovation of the error analysis consists in the occurance and treatment of a nonlinear reaction term $r(c)$ for the specie's concentration $c$ with unbounded derivative $r^{\prime}(\cdot)$. Only a Hölder condition is imposed on $r(\cdot)$. Moreover, to verify our model, a comparison of measurements and numerical calculations is done and the sensitivity of the mathematical model with respect to some input parameters that are material dependent and have to be prescribed is investigated. The sensitivity study is done by two- and three-dimensional computations.

The paper is organized as follows. Our mathematical model for enzymatic matrix degradation and drug delivery is introduced in Sect. 2. The discretization scheme that we use to solve the set of partial and ordinary differential equations is described and analyzed theoretically in Sect. 3. In Sect. 4, a computational investigation of the mathematical model is presented. In particular, the sensitivity of relevant quantities with respect to some model parameters is studied numerically and, moreover, the numerically calculated data are compared with experimentally determined data. Further, the convergence behaviour of the numerical discretization scheme is analyzed for a model problem. We end this work with some conclusions in Sect. 5.

\section{Mathematical model}

Drug release from dense collagen matrices as carrier systems for higher weight drugs is controlled by swelling and enzymatic degradation of the matrices; cf. $[8,15,16]$. Firstly, collagen is a hydrogel and swells after contact with aqueous solutions such that drug liberation by diffusion occurs. This mechanism was studied in a former work (cf. [18]) and is not considered here. Secondly, drug delivery systems containing collagen are degraded by collagenolytic enzymes such that the drug liberation becomes dependent on the enzyme binding and cleavage rate. This mechanism shall be described mathematically and simulated in the sequel. Separating the either mechanisms of drug release in their investigation, mathematical modelling and numerical simulation seems reasonable since they proceed on strongly different time scales, as our experimental studies have borne out; cf. [15]. Whereas drug release due to swelling is restricted to an initial period of some minutes (up to $30 \mathrm{~min}$ approximately), drug release by matrix degradation takes place over several days (up to 14 days possibly). The approach to separate the either mechanisms enabled us to apply more adapted mathematical and numerical methods to the separated subprocesses. An overall mathematical model combining the model components-drug release by swelling and drug release by matrix degradation - will be studied in a future work.

Therefore, from now on we assume a fully swollen carrier matrix along with an aqueous drug solution that is incorporated in the collagen fibre mesh. The hydrolytic degradation of a solid polymer matrix can occur by two extreme mechanisms. In the first one, refered to as heterogeneous, degradation is confined to the surface of the device and the undegraded carrier retains its chemical integrity during the process. In the other one, called homogeneous, hydrolysis involves random cleavage at a uniform rate throughout the bulk of the matrix. While the molecular weight of the polymer steadily decreases, the carrier can remain essentially intact until the polymer has undergone signifiant degradation, and reaches a critical molecular weight at which solubilization starts.

Based on our experimental observations (cf. $[15,16])$, we assume homogeneous degradation. Thus, the relevant processes to be described now are

- the diffusion of the enzyme in the swollen collagen matrix,

- the adsorption of the enzyme from the fluid to the collagen fibers,

- the enzymatic degradation of the polymer

- and, finally, the drug release.

The common reaction scheme for an enzymatically catalyzed degradation of a substrate was proposed by Michaelis and Menten (cf. [7]) and is given by

$\mathbf{E}+\mathbf{S} \stackrel{k_{1}}{\longrightarrow} \mathbf{E S}$

and

$\mathbf{E S} \stackrel{k_{2}}{\longrightarrow} \mathbf{E}+\mathbf{P}$,

where $\mathbf{E}$ is the enzyme, $\mathbf{S}$ the subtrate, $\mathbf{E S}$ the enzymesubstrate complex and $\mathbf{P}$ denotes the product. In the application under consideration, collagen represents the substrate whereas the hydrolyzed collagen is the product. Further, $k_{1}$ and $k_{2}$ denote rate parameters where $k_{1}$ describes the adsorption of enzyme onto the active binding site of the collagen fibre and $k_{2}$ characterizes the cleavage process. The first of the either reactions represents the adsorption process 
and the second one describes the cleavage of the substrate complex into a product and enzyme. The adsorption may be considered either as an equilibrium process or a kinetic one, depending on its time scale compared to the diffusion process. In the sequel, we will describe the adsorption of enzyme onto the collagen fibers as a kinetic reaction by means of a Freundlich-type isotherm which is based on our experimental investigations; cf. [15,16]. This is in agreement with [21], where also a Freundlich-type isotherm is proposed.

Assuming further that the diffusion of the enzyme in the swollen matrix is of Fickian type, our model equations describing the enzymatic collagen matrix degradation by means of (2.1), (2.2) then read as

$$
\begin{aligned}
\partial_{t} C_{E} & -\nabla \cdot\left(D_{E}\left(C_{K}\right) \nabla C_{E}\right)+k_{\mathrm{act}} C_{E} \\
& =-k_{1}\left(C_{E}\right)^{\alpha} C_{K}+k_{2} C_{E S}^{\gamma}, \\
\partial_{t} C_{E S} & =k_{1}\left(C_{E}\right)^{\alpha} C_{K}-k_{2} C_{E S}^{\gamma}, \\
\partial_{t} C_{K} & =-k_{1}\left(C_{E}\right)^{\alpha} C_{K},
\end{aligned}
$$

and

$\partial_{t} C_{P}-\nabla \cdot\left(D_{P} \nabla C_{P}\right)=k_{2} C_{E S}^{\gamma}$.

We consider solving (2.3)-(2.6), equipped with the initial conditions

$$
\begin{aligned}
& C_{E}(\cdot, 0)=0, \quad C_{E S}(\cdot, 0)=0, \\
& C_{K}(\cdot, 0)=C_{K}^{0}, \quad C_{P}(\cdot, 0)=0
\end{aligned}
$$

and the boundary conditions

$C_{E}(\cdot, t)=C_{E}^{\mathrm{ext}}, \quad C_{P}(\cdot, t)=0, \quad$ for $t>0$,

on $\Omega \times(0, T]$, where $\Omega \subset \mathbb{R}^{d}$ with $d=2$ or $d=3$ is the polymer matrix and $T>0$. Clearly, (2.7) is satisfied for all $\boldsymbol{x} \in \Omega$ and (2.8) for all $\boldsymbol{x} \in \Gamma=\partial \Omega$ where $\Gamma$ denotes the boundary of $\Omega$. In (2.3), (2.4), the variables $C_{E}, C_{E S}$ denote the concentrations, expressed in moles per volume, of the free enzyme and the enzyme-collagen complex, respectively. The variable $C_{P}$ denotes the concentration of the degradation product (hydrolyzed collagen), which is free to diffuse out of the polymer matrix. The enzyme activity decays in time which is incorporated in (2.3) by means of the term $k_{\text {act }} C_{E}$, with $k_{\text {act }}$ being a dimensionless constant that has to be determined experimentally. In (2.3)-(2.5), the quantity $\alpha$ denotes the parameter of the Freundlich isotherm and $k_{1}, k_{2}$ are the rate parameters of (2.1), (2.2). In particular, $\alpha \in(0,1]$ is satisfied. We note that in our simulation software a more general functional form is implemented for the rate $r$ describing the adsorption process (i.e., for the first term on the right-hand side of (2.3) and (2.4), respectively), namely, $r=-k_{1} \phi\left(C_{E}\right) C_{K}^{\delta}$ where $\phi(\cdot)$ can be chosen as a linear, Freundlich, Langmuir or Freundlich-Langmuir type isotherm and $\delta$ is a dimensionless parameter. In this paper, we use a Freundlich isotherm and set $\delta=1$, which is done due to our experimental oberservations; cf. [16]. Comparing our numerically calculated results with the measured data has shown that a nonlinear dependence of the right-hand side terms in (2.3)-(2.6) on $C_{E S}$ is necessary to describe adequately the enzymatic matrix degradation by the set of Eqs. (2.3)-(2.6). Thus, the parameter $\gamma>0$ is of empirical origin. In Sect. 4 , the sensitivity of the calculated results with respect to $\gamma$ is studied carefully.

In (2.7), $C_{K}^{0}$ denotes the collagen concentration at the initial time. In (2.8), $C_{E}^{\text {ext }}$ is the enzyme concentration in the ambient aqueous solution that has to be prescribed. Due to the degradation process occuring concurrently, the matrix phase through which the diffusion takes place changes continuously as a function of the extend of hydrolysis of the polymer. Therefore, the diffusion coefficient $D_{E}$ of the enzyme cannot be considered as a constant but rather has to be considered as a function of the fluid or collagen concentration. Here, we assume a Fujita-like dependence (cf. free volume theory in [9]) on the concentration of the collagen $C_{K}$, i.e., $D_{E}=D_{E}^{0} \exp \left(-\frac{\beta_{E} C_{K}}{C_{K}^{0}}\right)$, with $D_{E}^{0}$ denoting the diffusion coefficient of the enzyme in water and $\beta_{E}$ denoting a dimensionless constant. The diffusion coefficient $D_{P}$ of the product in the ambient fluid is assumed to be a constant which does not seem to be strongly limiting. The parameters $D_{E}^{0}$ and $D_{P}$ can be determined by measurements; cf. $[15,16]$.

A direct consequence of the assumption of homogeneous degradation and of the model Eqs. (2.3)-(2.8) is that the degradation of the polymeric substrate by the enzyme is independent of the active agent or drug, respectively. To model drug release, we assume (as also done, for instance, in [23]) that the initial load of active agent is composed of two pools: a pool of mobile active agent which is free to diffuse upon hydration of the matrix by the ambient aqueous solution, and some part which is immobilized by the polymer and can diffuse only after the degradation of the matrix. The immobilization of the active agent is due to physical entrapment only.

In our mathematical model we describe the release of the active agent by a diffusion equation with a source term that models the liberation of the immobilized active agent by matrix degradation. Precisely, the equation reads as

$\partial_{t} C_{A}-\nabla \cdot\left(D_{A}\left(C_{K}\right) \nabla C_{A}\right)=-\partial_{t} C_{A_{i}}$

where $C_{A}, C_{A_{i}}$ denote the concentrations of free and immobilized drug, respectively. We equip Eq. (2.9) with the initial and boundary condition

$$
\begin{aligned}
& C_{A}(\cdot, 0)=C_{A}^{0} \text { in } \Omega, \\
& C_{A}(\cdot, t)=0 \text { on } \Gamma, \text { for } t>0 .
\end{aligned}
$$

The initial concentration $C_{A}^{0}$ of the active agent that is free to diffuse can be determined experimentally by measuring 
the quantity of drug which would remain in the matrix if no degradation occurs, i.e., if no enzyme is available. To complete our model, a functional dependence of $C_{A_{i}}$ on $C_{A}$ is still needed. We assume that

$$
C_{A_{i}}=\sigma C_{K}^{\eta}
$$

where $\sigma \geq 0$ and $\eta>0$ are empirical parameters that have to be prescribed. In [23], it is simply assumed that $C_{A_{i}}=$ $\sigma C_{K}$ where $\sigma$ is a dimensionless constant that denotes the immobilizing capacity of the polymer and is equal to the number of hindering crosslinks or entanglements per mole of (fully swollen) substrate. The more general functional form (2.11) is based on our experimental observations; cf. [15, 16]. They have shown that the dependence of the released active agent on the collagen is nonlinear. The parameter $\eta$ is studied further in Sect. 4. Similarly to the enzyme, for the diffusion coefficient of the active agent in the matrix a functional form $D_{A}=D_{A}^{0} \exp \left(-\frac{\beta_{A} C_{K}}{C_{K}^{0}}\right)$ is used, where $D_{A}^{0}$ denotes the diffusion coefficient of the drug in the undegraded matrix, $C_{K}^{0}$ is the initial concentration of collagen and $\beta_{A}$ is a dimensionless constant; cf. [9,22].

Mathematically, we are thus faced with solving a nonlinear system of partial and ordinary differential equations that is given by (2.3)-(2.11). Concerning the existence and uniqueness of solutions of the Eqs. (2.3)-(2.11) the following result can directly be stated if the nonlinear dependence of the diffusion coefficients $D_{E}$ and $D_{A}$ on $C_{K}$ is neglected.

Theorem 1 Suppose that $\Omega \subset \mathbb{R}^{2}$ with $\partial \Omega \in C^{1,1}$. Let $p>2$ and $C_{A}^{0} \in W_{p}^{2-2 / p}(\Omega), C_{A}^{0} \geq 0$ in $\Omega$, and $C_{K}^{0} \in$ $C(\bar{\Omega}), C_{K}^{0} \geq 0$ in $\Omega$, be satisfied. Suppose that $C_{E}^{\mathrm{ext}} \in$ $W_{p}^{2-1 / p, 1-1 / 2 p}\left(\Gamma_{T}\right)$ and $C_{E}^{\mathrm{ext}} \geq 0$ on $\Gamma_{T}=\Gamma \times(0, T)$. Let $\alpha \in(0,1], \gamma \in(0,2]$ and $\eta>0$ be satisfied. Then, for any given $T \in(0, \infty)$ the system of Eqs. (2.3)-(2.11) admits an unique nonnegative solution

$$
\begin{aligned}
& C_{E} \in W_{p}^{2,1}\left(Q_{T}\right), C_{P} \in W_{p}^{2,1}\left(Q_{T}\right), C_{A} \in W_{p}^{2,1}\left(Q_{T}\right), \\
& p>2, C_{E S} \in C^{1}([0, T] ; C(\bar{\Omega})), C_{K} \in C^{1}([0, T] ; C(\bar{\Omega})) .
\end{aligned}
$$

In (2.12), we use the abbreviation $Q_{T}=\Omega \times(0, T)$. The notation of the space-time function spaces is standard; cf., e.g., [11]. We note that even the nonnegativeness of the species is ensured in Theorem 1. Essentially, Theorem 1 can be proved along the lines of [13], where for a model that is of a similar mathematical structure as the Eqs. (2.3)-(2.11) the existence and uniqueness of a solution is shown; cf. also $[4,14]$. The existence proof is based on the fixed-point theorem of Leray and Schauder. Restricting the range of the parameter $\gamma$ to the interval $(0,2]$ does not seem to be strongly limiting for our application; cf. Sect. 4. For a larger range of parameters $\gamma$ the local existence and uniqueness of a nonnegative solution to (2.3)-(2.11) can at least still be proved by a fixed-point argument of Schauder. Similarly, for the threedimensional case $\Omega \subset \mathbb{R}^{3}$ the local existence and uniquess of a nonnegative solution can be ensured.

\section{Discretization techniques}

We shall now describe briefly the discretization techniques that we apply to solve the Eqs. (2.3)-(2.11) numerically. Our numerical approach is based on the mixed finite element method (MFEM); cf. [5, 19]. We use a simulator that was originally developed at the Institute for Applied Mathematics of the University of Erlangen-Nürnberg for investigating reactive solute transport in the subsurface (cf. [19]) and was implemented in the software toolbox $u g$ (version 3.8); cf. [2].

More precisely, we use the backward Euler scheme for the temporal discretization of (2.3)-(2.11) and the lowest order Raviart-Thomas mixed finite elements for the spatial approximation; cf. [3,5,19]. Appreciable advantage of the MFEM is its local mass conservation property and that the fluxes over interelement boundaries remain continuous. Since the Eqs. (2.3)-(2.6) are coupled to each other, they are solved simultaneously by a damped version of Newton's method. For solving the linear system of the Newton iteration a multigrid algorithm is applied; cf. [3]. Since (2.3)-(2.6) do not depend on the active agent $C_{A}$, Eq. (2.9) is solved subsequently before proceeding to the next time step. Equation (2.9) could also be solved together with (2.3)-(2.6). The separation of (2.9) from (2.3)-(2.6) was only done for implementational reasons.

We shall now present briefly the algebraic systems that arise from discretizing (2.3)-(2.11) by the backward Euler scheme and the Raviart-Thomas lowest order mixed finite elements and need to be solved in each time step. Firstly, we rewrite the partial differential equations (2.3), (2.6) and (2.9) as first order systems of equations by introducing the flux variables $\boldsymbol{q}_{E}, \boldsymbol{q}_{P}$ and $\boldsymbol{q}_{A}$ of the free enzyme, the product and the active agent, respectively, as additional unknowns. Thus, we get that

$$
\begin{aligned}
& \partial_{t} C_{E}+\nabla \cdot \boldsymbol{q}_{E}+k_{\mathrm{act}} C_{E}=-R_{E}\left(C_{E}, C_{E S}, C_{K}\right), \\
& \boldsymbol{q}_{E}=-D_{E} \nabla C_{E}, \\
& \partial_{t} C_{P}+\nabla \cdot \boldsymbol{q}_{P}=R_{P}\left(C_{E S}\right), \\
& \boldsymbol{q}_{P}=-D_{P} \nabla C_{P}, \\
& \partial_{t} C_{A}+\nabla \cdot \boldsymbol{q}_{A}=\sigma \eta C_{K}^{(\eta-1)} R_{K}\left(C_{E}, C_{K}\right), \\
& \boldsymbol{q}_{A}=-D_{A}\left(C_{K}\right) \nabla C_{A},
\end{aligned}
$$


and from (2.4), (2.5)

$\partial_{t} C_{E S}=R_{E}\left(C_{E}, C_{E S}, C_{K}\right)$,

$\partial_{t} C_{K}=-R_{K}\left(C_{E}, C_{K}\right)$,

where

$R_{K}\left(C_{E}, C_{K}\right)=k_{1}\left(C_{E}\right)^{\alpha} C_{K}$,

$R_{P}\left(C_{E S}\right)=k_{2} C_{E S}^{\gamma}$,

$R_{E}=R_{K}\left(C_{E}, C_{K}\right)-R_{P}\left(C_{E S}\right)$.

In the sequel we need further notations. Let $t_{0}<t_{1}<\cdots<$ $t_{N}$ with $t_{0}=0$ and $t_{N}=T$ denote the grid for the time variable $t$ on the interval $[0, T]$ with step size $\tau_{n}=t_{n}-$ $t_{n-1} . L^{2}(\Omega)$ and $\boldsymbol{L}^{2}(\Omega)$ are the spaces of all strongly measurable, square integrable, scalar-valued and vector-valued functions on $\Omega$, respectively, and $\boldsymbol{H}(\operatorname{div} ; \Omega)$ is the space of all $\boldsymbol{L}^{2}$-vector functions with divergence in $L^{2}(\Omega)$. Let $\mathcal{T}_{h}=\{T\}$ be a decomposition of $\Omega \subset \mathbb{R}^{d}$ into triangles or tetrahedrons, respectively. The decomposition is assumed to be face to face. By $\mathcal{S}_{h}$ we denote the set of all faces of $\mathcal{T}_{h}$. We compute approximations of the variables in the $L B B$-stable pair of finite element spaces $W_{h} \times \boldsymbol{V}_{h} \subset L^{2}(\Omega) \times \boldsymbol{H}(\operatorname{div} ; \Omega)$, where

$W_{h}=\left\{w_{h} \in L^{2}(\Omega) \mid w_{h}\right.$ is constant on $\left.T, \forall T \in \mathcal{T}_{h}\right\}$

and

$$
\begin{gathered}
\boldsymbol{V}_{h}=\left\{\boldsymbol{q} \in \boldsymbol{H}(\operatorname{div} ; \Omega) \mid \boldsymbol{q}(\boldsymbol{x})_{\mid T}=\boldsymbol{a}+b \boldsymbol{x},\right. \\
\left.\boldsymbol{x} \in T, \mathbf{a} \in \mathbb{R}^{d}, \quad b \in \mathbb{R}, \quad \forall T \in \mathcal{T}_{h}\right\} .
\end{gathered}
$$

$\boldsymbol{V}_{h}$ is the lowest order Raviart-Thomas space; cf. [5]. Consequently, for the concentrations of the free enzyme, the enzyme-substrate complex, the collagen, the product and the active agent we consider determining approximations $C_{\mathrm{ET}}, C_{\mathrm{EST}}, C_{\mathrm{KT}}, C_{\mathrm{PT}}$ and $C_{\mathrm{AT}}$ that are constant on each element $T$. This yields one degree of freedom per specie and element. The solutions of the ordinary differential equations (2.4) and (2.5) are thus approximated also in $W_{h}$. The degrees of freedom of fluxes $\boldsymbol{v}_{h} \in \boldsymbol{V}_{h}$ are their normal components $\boldsymbol{v}_{h} \cdot \boldsymbol{v}$ on the faces $S \in \mathcal{S}_{h}$. Hence, for the fluxes $\boldsymbol{q}_{i}, i=$ $E, P, A$, we get one degree of freedom $q_{i, S}, i=E, P, A$, on each face $S \in \mathcal{S}_{h}$. For further characterizations of the spaces $W_{h}$ and $\boldsymbol{V}_{h}$ we refer to [5].

However, applying the mixed finite element method in its dual formulation to the time discrete versions of (3.1)(3.7) leads to indefinite systems of equations such that standard iterative linear solvers cannot be applied; cf. [5] for the linear case and $[3,19]$ for nonlinear problems. To overcome this drawback of the MFEM, we use a hybrid variant of the approach by introducing Lagrange multipliers; cf. [5]. For the application of this technique in a nonlinear framework we refer, in particular, to $[3,19]$. The Lagrange multiplier technique allows us to relax the continuity constraint on the normal components of the fluxes over interelement faces, which is implied by $\boldsymbol{q}_{i} \in \boldsymbol{H}(\operatorname{div} ; \Omega)$ for $i=E, P, A$, to requiring that only $\boldsymbol{q}_{i} \in H(\operatorname{div} ; T)$ is satisfied for all $T \in \mathcal{T}_{h}$. Then, the continuity of the normal fluxes is respectively ensured by an additional variational equation involving the Lagrange multipliers $\lambda_{E}, \lambda_{P}$ and $\lambda_{A}$ that are defined on the faces and are piecewise constant there, i.e., $\lambda_{E}, \lambda_{P}, \lambda_{A} \in$ $\Lambda_{h}=\left\{\lambda \in L^{2}\left(\mathcal{S}_{h}\right) \mid \lambda_{\mid S}=\right.$ constant $\left.\forall S \in \mathcal{S}_{h}\right\}$. After an elimination of internal degrees of freedom, also known as static condensation, the degrees of freedom $\lambda_{E S}, \lambda_{P S}$ and $\lambda_{A S}$ of the Lagrange multipliers become the unknowns of a global nonlinear system of equations which we solve by Newton's method and a multigrid algorithm as mentioned before; cf. [3,19] for technical details. Precisely, for each of the species enzyme, product and active agent we get one degree of freedom per face which can also be considered as an approximation of the concentration profile on the interelement face and, moreover, as a degree of freedom of a second order accurate nonconforming Crouzeix-Raviart discretization of the concentratio $\mathrm{n}$ profile; $\mathrm{cf}$. [5] for details.

Then the nonlinear algebraic problem that has to be solved at time $t_{n}, n=1, \ldots, N$, for the transport of enzyme and the collagen matrix degradation reads as follows: for given $C_{\mathrm{ET}}^{n-1}$, $C_{\mathrm{EST}}^{n-1}, C_{\mathrm{KT}}^{n-1}, C_{\mathrm{PT}}^{n-1}$ find $C_{\mathrm{ET}}^{n}, C_{\mathrm{EST}}^{n}, C_{\mathrm{KT}}^{n}, C_{\mathrm{PT}}^{n}, \lambda_{E S}^{n}, \lambda_{P S}^{n}$ and $q_{E, T S}^{n}, q_{P, T S}^{n}$ such that it holds that

$$
\begin{aligned}
& C_{\mathrm{ET}}^{n}+\frac{\tau_{n}}{V_{T}} \sum_{S \subset T} q_{E, T S}^{n}+\tau_{n} k_{\mathrm{act}} C_{\mathrm{ET}}^{n} \\
& =C_{\mathrm{ET}}^{n-1}-\tau_{n} R_{E}\left(C_{\mathrm{ET}}^{n}, C_{\mathrm{EST}}^{n}, C_{\mathrm{KT}}^{n}\right), \\
& C_{\mathrm{EST}}^{n}-C_{\mathrm{EST}}^{n-1}=\tau_{n} R_{E}\left(C_{\mathrm{ET}}^{n}, C_{\mathrm{EST}}^{n}, C_{\mathrm{KT}}^{n}\right), \\
& C_{\mathrm{KT}}^{n}-C_{\mathrm{KT}}^{n-1}=-\tau_{n} R_{K}\left(C_{\mathrm{ET}}^{n}, C_{\mathrm{KT}}^{n}\right), \\
& C_{\mathrm{PT}}^{n}+\frac{\tau_{n}}{V_{T}} \sum_{S \subset T} q_{P, T S}^{n}=C_{\mathrm{PT}}^{n-1}+\tau_{n} R_{P}\left(C_{\mathrm{EST}}^{n}\right), \\
& \sum_{S^{\prime} \subset T} B_{T S S^{\prime}} q_{E, T S^{\prime}}^{n}=D_{E}\left(C_{\mathrm{ET}}^{n}-\lambda_{E S}^{n}\right), \\
& \sum_{S^{\prime} \subset T} B_{T S S^{\prime}} q_{P, T S^{\prime}}^{n}=D_{P}\left(C_{\mathrm{PT}}^{n}-\lambda_{P S}^{n}\right), \\
& q_{E, T S}^{n}=-q_{E, T^{\prime} S}^{n}, \\
& q_{P, T S}^{n}=-q_{P, T^{\prime} S}^{n} .
\end{aligned}
$$

Equations (3.11)-(3.14) have to be satisfied for all $T \in \mathcal{T}_{h}$, Eqs. (3.15), (3.16) have to be fulfilled for all $T \in \mathcal{T}_{h}$ and $S \subset \partial T$ and, finally, the continuity constraints (3.17), (3.18) are required for all $S \in \mathcal{S}_{h}$ where $S \subset \partial T$ and $S \subset \partial T^{\prime}$ is assumed. In (3.11)-(3.18), we equipped the degrees of freedom of the flux variables with an additional index $T$, i.e., we write $q_{i, T S}^{n}$ instead of $q_{i, S}^{n}$, for $i=E, P$. This is only done for the hybridization of the mixed finite approximation of (3.1), (3.3) and the resulting elementwise formulation of 
the constraints. In (3.11)-(3.16), $V_{T}$ denotes the volume of the element $T$ and

$B_{T S S^{\prime}}=\int_{T} \boldsymbol{v}_{T S} \cdot \boldsymbol{v}_{T S^{\prime}} d \boldsymbol{x}$,

where $\boldsymbol{v}_{T S}, \boldsymbol{v}_{T S^{\prime}}$ are the local basis functions of the lowest order Raviart-Thomas space on the element $T$ with respect to the faces $S$ and $S^{\prime}$; cf. [3,5]. The Dirichlet boundary conditions (2.8) are incorporated in the discrete scheme (3.11)(3.18) in the usual way by putting $\lambda_{i, S}^{n}=|E|^{-1} \int_{E} C_{i}\left(\cdot, t_{n}\right)$ $d \sigma$ for $E \subset \Gamma, i=E, P$; cf. [3,5,19]. For details of the elimation of internal degrees of freedom in (3.11)-(3.18) and the solution of resulting nonlinear global system for the Lagrange multipliers by Newton's method we refer to the literature; cf. [3,19].

Similarly, once the collagen concentration at time $t_{n}, n=$ $1, \ldots, N$ has been computed, the drug concentration profile at $t_{n}$ is calculated by solving the following discrete problem: for given $C_{\mathrm{AT}}^{n-1}, C_{\mathrm{ET}}^{n}, C_{\mathrm{KT}}^{n}$ find $C_{\mathrm{AT}}^{n}, \lambda_{A S}^{n}$ and $q_{A, T S}^{n}$ such that it holds that

$$
\begin{aligned}
& C_{\mathrm{AT}}^{n}-C_{\mathrm{AT}}^{n-1}+\frac{\tau_{n}}{V_{T}} \sum_{S \subset T} q_{A, T S}^{n} \\
& \quad=\tau_{n} \sigma \eta C_{\mathrm{KT}}^{n}{ }^{(\eta-1)} R_{K}\left(C_{\mathrm{ET}}^{n}, C_{\mathrm{KT}}^{n}\right), \\
& \sum_{S^{\prime} \subset T} B_{T S S^{\prime}} q_{A, T S^{\prime}}^{n} \\
& \quad=D_{A}\left(C_{\mathrm{KT}}^{n}\right)\left(C_{\mathrm{AT}}^{n}-\lambda_{A S}^{n}\right), \\
& q_{A, T S}^{n}=-q_{A, T^{\prime} S}^{n} .
\end{aligned}
$$

Again, the Eqs. (3.19)-(3.21) are to be satisfied for all $T \in$ $\mathcal{T}_{h}$, for all $T \in \mathcal{T}_{h}, S \subset \partial T$ and for all $S \in \mathcal{S}_{h}$ with $S \subset \partial T$, $S \subset \partial T^{\prime}$, respectively.

A crucial point in the numerical analysis of the discretization scheme (3.11)-(3.18) is the occurance of reaction terms that are of the form $C_{i}^{\alpha}, i=E, E S, K$, with a parameter $\alpha$ that may be less than one, i.e., $\alpha \in(0,1)$. For functions $r(c)=c^{\alpha}$ with $\alpha \in(0,1)$ the derivatives $r^{\prime}(c)=\alpha c^{\alpha-1}$ become unbounded if $c$ converges to 0 which makes difficulties in the error analysis, as we shall see below. Parameters $\alpha \geq 1$ are less crucial in this respect and, therefore, not studied here. Thus, in the sequel we shall show for the semilinear model problem

$-\Delta c+r(c)=f \quad$ in $\Omega, \quad c=0 \quad$ on $\partial \Omega$

that under the assumptions that

$$
r \in C^{1} \text { is nondecreasing and }
$$

$\left|r\left(c_{1}\right)-r\left(c_{2}\right)\right| \leq L\left|c_{1}-c_{2}\right|^{\alpha}$

is satisfied for some parameter $\alpha \in(0,1)$, the convergence of the lowest order Raviart-Thomas mixed finite element approximation is ensured. But, the convergence is only suboptimal of the order $\frac{1+\alpha}{2}$ instead of the order 1. In (3.22), $\Omega \subset \mathbb{R}^{d}$ is a bounded domain with Lipschitz-continuous boundary $\partial \Omega$. Considering the steady model problem (3.22) instead of the full set of equations, allows us to avoid technical overhead and reduce the error analysis to its essential elements. By the techniques developed in [20] the error analysis can be extended easily to the unsteady counterpart of problem (3.22).

Analyzing the mixed finite element discretization of problem (3.22)-(3.24) differs from former publications insofar as we impose here weaker assumptions on the parametrization $r(\cdot)$ of the reaction term. Only the boundedness of $r(\cdot)$ and its Hölder continuity (3.24) is assumed. For instance, Chen [6] analyses an expanded MFEM under the assumption that the parametrization of the reaction term is twice continuously differentiable with bounded derivatives through second order. However, this condition does not apply to our model (2.3)-(2.11) with the reaction term being defined by (3.8) with $\alpha \in(0,1)$.

The mixed finite element approximation of (3.22) then reads as follows: for given $f \in L^{2}(\Omega)$ find $\{c, \boldsymbol{q}\} \in$ $L^{2}(\Omega) \times \boldsymbol{H}(\operatorname{div} ; \Omega)$ such that it holds that

$\langle\nabla \cdot \boldsymbol{q}, w\rangle+\langle r(c), w\rangle=\langle f, w\rangle$,

$\langle\boldsymbol{q}, \boldsymbol{v}\rangle-\langle c, \nabla \cdot \boldsymbol{v}\rangle=0$

for all $w \in L^{2}(\Omega)$ and $\boldsymbol{v} \in \boldsymbol{H}(\operatorname{div} ; \Omega)$.

The corresponding discrete variational formulation reads as follows: for given $f \in L^{2}(\Omega)$ find $\left\{c_{h}, \boldsymbol{q}_{h}\right\} \in W_{h} \times \boldsymbol{V}_{h}$ such that it holds that

$\left\langle\nabla \cdot \boldsymbol{q}_{h}, w_{h}\right\rangle+\left\langle r\left(c_{h}\right), w_{h}\right\rangle=\left\langle f, w_{h}\right\rangle$,

$\left\langle\boldsymbol{q}_{h}, \boldsymbol{v}_{h}\right\rangle-\left\langle c_{h}, \nabla \cdot \boldsymbol{v}_{h}\right\rangle=0$

for all $w_{h} \in W_{h}$ and $\boldsymbol{v}_{h} \in \boldsymbol{V}_{h}$.

In the sequel, we denote by $P_{h}: L^{2}(\Omega) \mapsto W_{h}$ and $\Pi_{h}$ : $\boldsymbol{H}($ div; $\Omega) \mapsto \boldsymbol{V}_{h}$, the standard interpolation operators; cf. [17]. In particular, $P_{h}$ and $\boldsymbol{\Pi}_{h}$ satisfy

$$
\begin{aligned}
& \left\|w-P_{h} w\right\|_{L^{2}(\Omega)} \leq C h\|w\|_{H^{1}(\Omega)}, \\
& \left\|\boldsymbol{v}-\boldsymbol{\Pi}_{h} \boldsymbol{v}\right\|_{\boldsymbol{L}^{2}(\Omega)} \leq C h\|\boldsymbol{v}\|_{\boldsymbol{H}^{1}(\Omega)}
\end{aligned}
$$

for all $w \in H^{1}(\Omega)$ and $\boldsymbol{v} \in \boldsymbol{H}^{1}(\Omega)$, respectively. The definition of the spaces $H^{1}(\Omega)$ and $\boldsymbol{H}^{1}(\Omega)$ is standard; cf., e.g., $[1,5]$.

For the scheme (3.27) and (3.28) the following error estimate holds.

Theorem 2 Suppose that $(c, \boldsymbol{q}) \in W \times \boldsymbol{V}$ solves (3.25) and (3.26). Let $\left(c_{h}, \boldsymbol{q}_{h}\right) \in W_{h} \times \boldsymbol{V}_{h}$ satisfy (3.27) and (3.28). 
Then we have that

$\left\langle r(c)-r\left(c_{h}\right), c-c_{h}\right\rangle+\left\|\boldsymbol{q}-\boldsymbol{q}_{h}\right\|_{\boldsymbol{L}^{2}(\Omega)}^{2} \leq C\left(h^{2}+h^{1+\alpha}\right)$,

with $C$ denoting a generic constant, not depending on the mesh size $h$.

Proof Substracting (3.27) and (3.28) from (3.25) and (3.26), respectively, we obtain that

$$
\begin{aligned}
& \left\langle\nabla \cdot\left(\boldsymbol{q}-\boldsymbol{q}_{h}\right), w_{h}\right\rangle+\left\langle r(c)-r\left(c_{h}\right), w_{h}\right\rangle=0, \\
& \left\langle\boldsymbol{q}-\boldsymbol{q}_{h}, \boldsymbol{v}_{h}\right\rangle-\left\langle c-c_{h}, \nabla \cdot \boldsymbol{v}_{h}\right\rangle=0
\end{aligned}
$$

for all $w_{h} \in W_{h}$ and $\boldsymbol{v}_{h} \in \boldsymbol{V}_{h}$, respectively. We recall that $\nabla \cdot \boldsymbol{v}_{h} \in W_{h}$ such that $\left\langle c-c_{h}, \nabla \cdot \boldsymbol{v}_{h}\right\rangle=\left\langle P_{h} c-c_{h}, \nabla \cdot\right.$ $\left.\boldsymbol{v}_{h}\right\rangle$. Further, by the definition of $\boldsymbol{\Pi}_{h}$, it holds that $\langle\nabla \cdot(\boldsymbol{q}-$ $\left.\left.\boldsymbol{q}_{h}\right), w_{h}\right\rangle=\left\langle\nabla \cdot\left(\boldsymbol{\Pi} \boldsymbol{q}-\boldsymbol{q}_{h}\right), w_{h}\right\rangle$. Then, choosing $w_{h}=$ $P_{h} c-c_{h}$ and $\boldsymbol{v}_{h}=\boldsymbol{\Pi}_{h} \boldsymbol{q}-\boldsymbol{q}_{h}$ and adding the resulting equations we get that

$$
\left\langle r(c)-r\left(c_{h}\right), P_{h} c-c_{h}\right\rangle+\left\langle\boldsymbol{q}-\boldsymbol{q}_{h}, \boldsymbol{\Pi}_{h} \boldsymbol{q}-\boldsymbol{q}_{h}\right\rangle=0 .
$$

From (3.33) we deduce that

$$
\begin{aligned}
& \left\langle r(c)-r\left(c_{h}\right), c-c_{h}\right\rangle+\left\|\boldsymbol{q}-\boldsymbol{q}_{h}\right\|_{\boldsymbol{L}^{2}(\Omega)}^{2} \\
& \quad=\left\langle\boldsymbol{q}-\boldsymbol{q}_{h}, \boldsymbol{q}-\boldsymbol{\Pi}_{h} \boldsymbol{q}\right\rangle+\left\langle r(c)-r\left(c_{h}\right), c-P_{h} c\right\rangle .
\end{aligned}
$$

Since $r(\cdot)$ is a nondecreasing function by means of (3.23), the first of the either terms on the left-hand side of (3.34) is positive. By (3.24) it holds that

$$
\begin{aligned}
& \left\langle r(c)-r\left(c_{h}\right), c-c_{h}\right\rangle \geq \frac{1}{2}\left\langle r(c)-r\left(c_{h}\right), c-c_{h}\right\rangle \\
& +\frac{1}{2 L^{\frac{1}{\alpha}}}\left\|r(c)-r\left(c_{h}\right)\right\|_{L^{\frac{1+\alpha}{\alpha}}(\Omega)}^{\frac{1+\alpha}{\alpha}} .
\end{aligned}
$$

In (3.35), $L$ is the constant of the Hölder-continuity condition (3.24). For the first of the either terms on the right-hand side of (3.34) we have that

$$
\begin{aligned}
& \left\langle\boldsymbol{q}-\boldsymbol{q}_{h}, \boldsymbol{q}-\boldsymbol{\Pi}_{h} \boldsymbol{q}\right\rangle \leq \frac{1}{2}\left\|\boldsymbol{q}-\boldsymbol{q}_{h}\right\|_{\boldsymbol{L}^{2}(\Omega)}^{2} \\
& +\frac{1}{2}\left\|\boldsymbol{q}-\boldsymbol{\Pi}_{h} \boldsymbol{q}\right\|_{\boldsymbol{L}^{2}(\Omega)}^{2} .
\end{aligned}
$$

Using the Cauchy-Young inequality, i.e., $a b \leq p^{-1} a^{p}+$ $q^{-1} b^{q}$ for $a, b>0$ and $p, q \in(1, \infty)$ with $p^{-1}+q^{-1}=1$, with $a=\delta\left|r(c)-r\left(c_{h}\right)\right|, b=\delta^{-1}\left|c-P_{h} c\right|$ and $p=(1+$ $\alpha) \alpha^{-1}$ we observe that the second of the either right-hand side terms in (3.34) is bounded through

$$
\begin{aligned}
\langle r(c)- & \left.r\left(c_{h}\right), c-P_{h} c\right\rangle \\
= & \int_{\Omega}\left(r(c)-r\left(c_{h}\right)\right)\left(c-P_{h} c\right) d \boldsymbol{x} \\
\leq & \frac{\alpha \delta^{\frac{1+\alpha}{\alpha}}}{1+\alpha} \int_{\Omega}\left|r(c)-r\left(c_{h}\right)\right|^{\frac{1+\alpha}{\alpha}} d \boldsymbol{x} \\
& +\frac{\delta^{-(1+\alpha)}}{1+\alpha} \int_{\Omega}\left|c-P_{h} c\right|^{1+\alpha} d \boldsymbol{x} \\
\leq & \frac{\alpha \delta^{\frac{1+\alpha}{\alpha}}}{1+\alpha}\left\|r(c)-r\left(c_{h}\right)\right\|_{L^{\frac{1+\alpha}{\alpha}}(\Omega)}^{\frac{1+\alpha}{1+\alpha}} \\
& +\frac{\delta^{-(1+\alpha)}}{1+\alpha}\left\|c-P_{h} c\right\|_{L^{1+\alpha}(\Omega)}^{1+\alpha} .
\end{aligned}
$$

By a suitable choice of $\delta$ we then obtain from (3.34)-(3.37) that

$$
\begin{aligned}
& \left\langle r(c)-r\left(c_{h}\right), c-c_{h}\right\rangle+\left\|\boldsymbol{q}-\boldsymbol{q}_{h}\right\|_{\boldsymbol{L}^{2}(\Omega)}^{2} \\
& \quad \leq C\left\|\boldsymbol{q}-\boldsymbol{\Pi}_{h} \boldsymbol{q}\right\|_{\boldsymbol{L}^{2}(\Omega)}^{2}+C\left\|c-P_{h} c\right\|_{L^{1+\alpha}(\Omega)}^{1+\alpha} .
\end{aligned}
$$

Finally, we combine (3.38) with (3.29) and use the Sobolev embedding of $L^{2}(\Omega)$ in $L^{1+\alpha}(\Omega)$ for $\alpha \in(0,1)$; cf. [1, p. 25, Theorem 2.8]. This proves the assertion (3.30).

We note that (3.30) along with (3.35) implies that $\left\|r(c)-r\left(c_{h}\right)\right\|_{L^{\frac{1+\alpha}{\alpha}}(\Omega)}^{\frac{1+\alpha}{\alpha}}+\left\|\boldsymbol{q}-\boldsymbol{q}_{h}\right\|_{\boldsymbol{L}^{2}(\Omega)}^{2} \leq C\left(h^{2}+h^{1+\alpha}\right)$.

\section{Parameter sensitivity studies and computational investigations}

In this section we shall present some of our numerical results and investigations to demonstrate the capability of the mathematical model to describe reliably enzymatically catalyzed degradation of collagen matrices and simultaneous drug delivery. In particular, we shall analyze the sensitivity of the calculated characteristic quantities with respect to the model parameters $\alpha$ and $\gamma$. These crucial parameters characterize the degree of nonlinearity in the binding of the enzyme at the collagen substrate and in the degradation of the collagen by cleavage of the fibers. Further, we investigate numerically the influence of the matrix dimension on the enzymatic degradation and drug release process. Cylindrical matrices are considered for which two and three dimensional computations were performed. The set of parameters that was used for the calculations is summarized in Table 1. Most of these parameters were determined by measurements. The values given in Table 1 for $\alpha, \gamma$ and $\eta$ were determined in [16] by least-squares fitting and have to be considered as best-fit numbers. For further details, also for a precise characterization of the compounds (collagenous material and drug FITC 
Table 1 Parameters of the numerical simulations; cf. [16]

\begin{tabular}{llllll}
\hline Collagen degradation & \multicolumn{3}{l}{ Drug release } \\
\hline$R$ & $0.139 \mathrm{~cm}$ & $D_{E}^{0}$ & $2.410^{-3} \mathrm{~cm}^{2} / \mathrm{h}$ & $D_{A}^{0}$ & $1.3710^{-3} \mathrm{~cm}^{2} / \mathrm{h}$ \\
$\beta_{E}$ & 0.5 & $D_{P}$ & $2.410^{-3} \mathrm{~cm}^{2} / \mathrm{h}$ & $\beta_{A}$ & 0.5 \\
$C_{K}^{0}$ & $1.12 \mu \mathrm{mol} / \mathrm{cm}^{3}$ & $C_{E}^{\text {ext }}$ & $1.410^{-6} \mu \mathrm{mol} / \mathrm{cm}^{3}$ & $C_{A}^{0}$ & 0 \\
$k_{\text {act }}$ & 0 & $k_{1}$ & $4579 \mathrm{~cm}^{3} /(\mu \mathrm{mol} \mathrm{h})$ & $C_{A i}^{0}$ & $1.36 \mu \mathrm{mol}^{0} \mathrm{~cm}^{3}$ \\
$k_{2}$ & $1741 / \mathrm{h}$ & $\alpha$ & 0.673 & $\sigma$ & $C_{A i}^{0} /\left(C_{K}^{0}\right)^{\eta}$ \\
$\gamma$ & 0.44 & & & $\eta$ & 1.5 \\
\hline
\end{tabular}

Fig. 1 Sensitivity analysis for the model parameters $\alpha$ (left) and $\gamma$ (right)
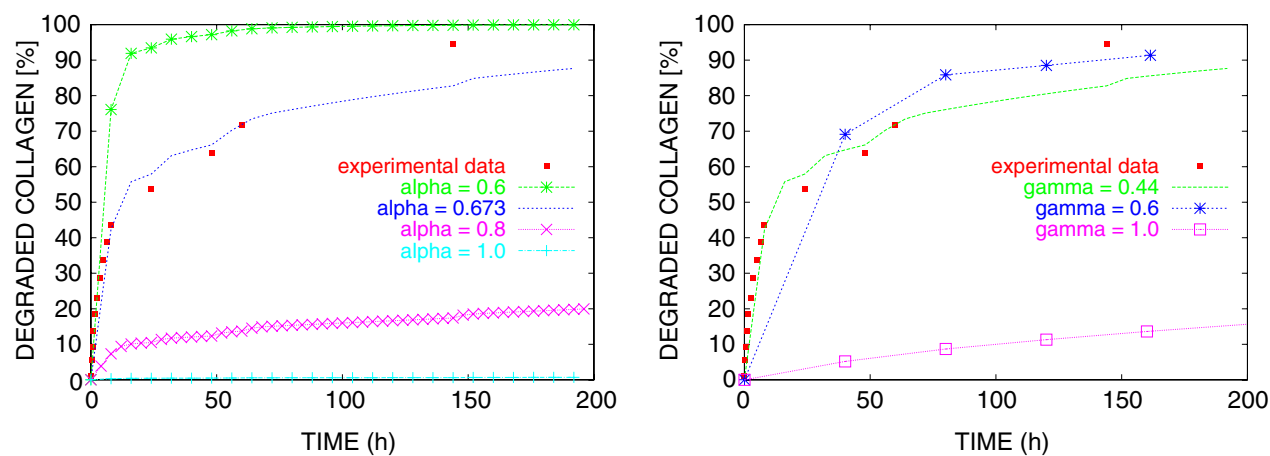

dextran), we refer to [16]. Finally, the spatial convergence behaviour of the mixed finite element discretization is investigated for the model problem (3.22). This is done in order analyse the sharpness of the error estimate (3.30). We note that the numerical simulations that are presented in the sequel cover only a small range of our performed parameter sensitivity and model validation studies.

We first analyze the sensitivity of our model with respect to the parameter $\alpha$. Simulations were done for $\alpha=$ $0.6,0.673,0.8,1.0$. The calculated percentage of degraded collagen versus time is visualized in the left plot of Fig. 1. The experimentally measured data (cf. [16] for details) are given by the points with red squares. We conclude from this plot that even small variations of the value of $\alpha$ lead to completely different collagen degradation profiles. Thus, our model is sensitive with respect to the parameter $\alpha$ that should be specified carefully for the respectively used collagenous material. Moreover, the curves show that the simplest choice $\alpha=1$ does not lead to a sufficiently accurate description of the degradation process.

Next, we study the sensitivity of our model with respect to the parameter $\gamma$ characterizing the degree of nonlinearity in the cleavage process. In the right plot of Fig. 1 the calculated percentage of degraded collagen versus time is visualized for $\gamma=0.44,0.6,1.0$. In the literature (cf. [10, 12,23,24]), the parameter $\gamma$ is usually chosen equal to 1.0. However, there is no experimental evidence for this choice. On the contrary, our experimental results (cf. Fig. 1) show that the degradation of collagen runs much faster at the initial stage, when the enzyme concentration in the matrix is still relatively low. This observation actually motivated us to describe the cleavage of the collagen fibers in terms of a nonlinear model by equipping $C_{E S}$ on the right-hand sides of (2.3), (2.4) and (2.6) with an extra power $\gamma$ in order to get more flexibility. In the right plot of Fig. 1 we observe that the slopes of the curves change substantially under variation of $\gamma$. Thus, this parameter also has to be chosen carefully for the respectively used collagenous material.

The next study is devoted to the influence of the radius and of the height of a bacillary drug delivery system on the collagen degradation and drug release process. We performed both, two and three dimensional simulations. The reference matrix has a radius of $0.139 \mathrm{~cm}$ and a height of $1.35 \mathrm{~cm}$ which were determined by measurements; cf. [16]. First, we performed two-dimensional simulations by considering a transversal cross section of the cylindrical matrix. Computations were done for the radius being equal to $0.139 \mathrm{~cm}$, for halved radius and, finally, for doubled radius. The calculated percentage of degraded collagen and released drug are visualized in Fig. 2. Eventhough the differences between the considered matrix systems are not really large, we note that at least at the initial stage a smaller radius leads to a faster degradation of the collagen matrix and a faster drug delivery which is an important qualitative observation for the design, optimization and in vivo application of collagenous drug delivery systems.

Next, we analyze the impact of the matrix height on the degradation and release profiles. Three-dimensional simulations were done for matrices of radius equal to $0.139 \mathrm{~cm}$ and of height equal to $1.35,2.27$ and $4.54 \mathrm{~cm}$. The calculated results are visualized in the left plot of Fig. 3. A significant 
Fig. 2 Sensitivity analysis for the radius of the matrix: two (left) and three (right) dimensional simulations
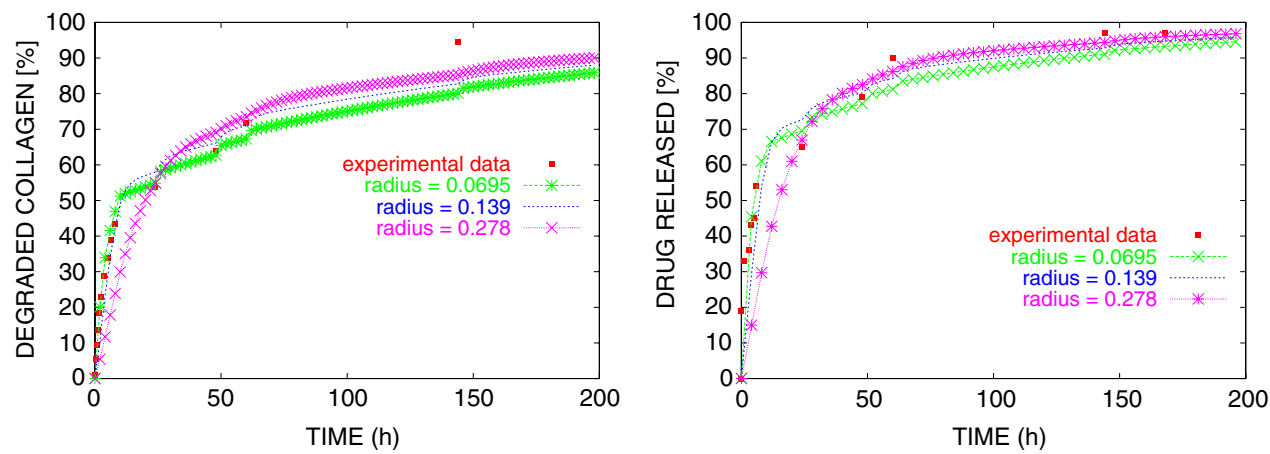

Fig. 3 Sensitivity analysis for the height of the matrix by three dimensional computations (left) and comparison with the two-dimensional results (right)
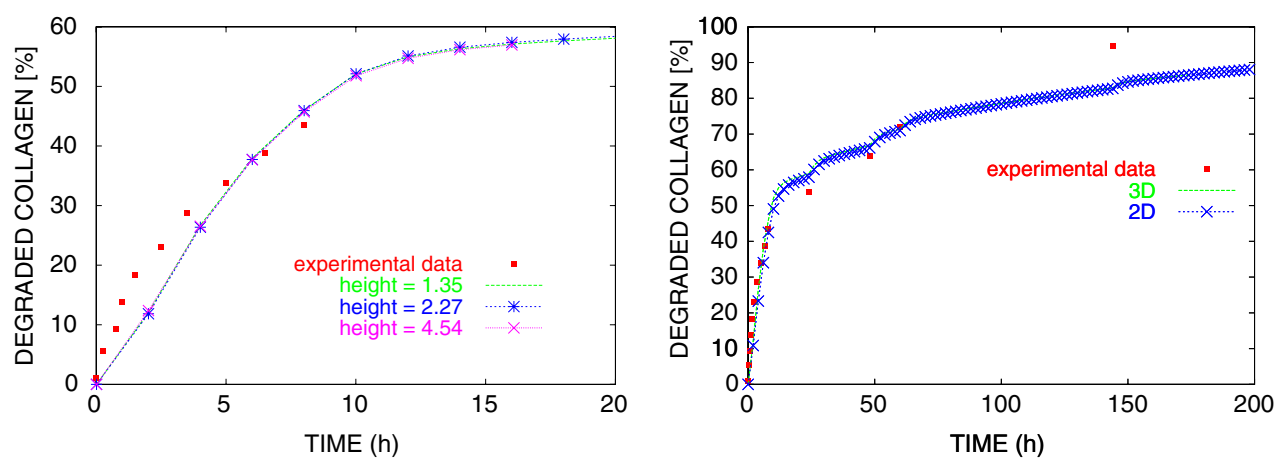

dependence of the degradation profile on the matrix height is not observed. Moreover, the three-dimensional calculation predicts almost the same degradation profiles for the collagen matrix as they are obtained by two-dimensional calculations. This is shown in the right plot of Fig. 3. Thus, the matrix height and longitudinal effects do not seem to perturb the collagen degradation and drug delivery significantly. The degradation and release profiles that we obtained by threedimensional computations for halved and doubled radius are almost the same as in the two-dimensional case (cf. Fig. 2) and, therefore, not given here.

The last sensitivity study is concerned with the influence of the parameter $\eta$ on the drug release profile. This parameter describes the nonlinear dependence of the immobilized concentration of drug on the concentration of collagen [cf. (2.11)]. In practice, the parameter $\eta$ has to be fixed for the respective collagenous material by least-squares fitting. For the considered material (cf. Table 1) the best parameter fit was obtained for $\eta=1.5$; cf. [16]. In Fig. 4 a relatively strong sensitivity of the calculated drug release profile with respect to the model parameter $\eta$ is observed. Increasing numbers of $\eta$ lead to a faster drug release; cf. Fig. 4.

Finally, for illustration purposes the calculated collagen and drug concentration profiles of a two-dimensional simulation (transversal cross section of a bacillary drug delivery system) are visualized in Fig. 5. For the computations we used the parameter set that is given in Table 1 .

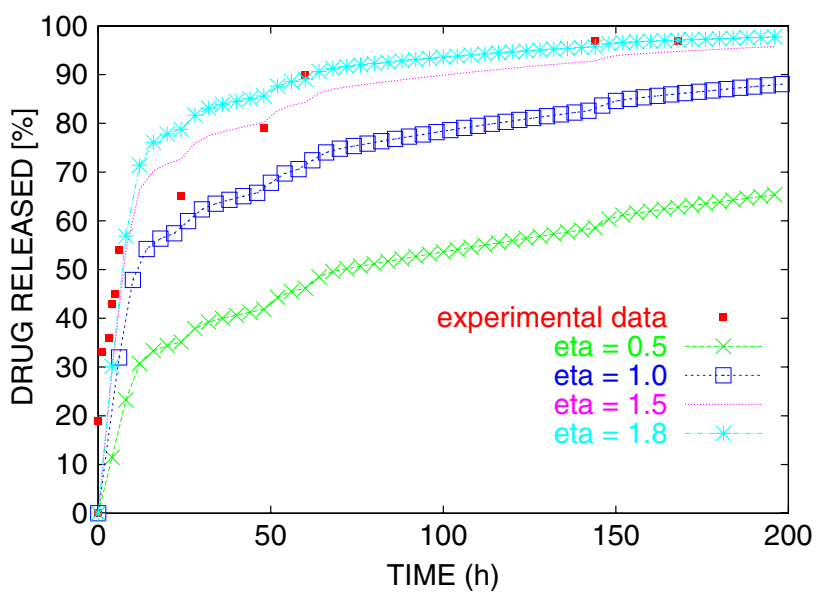

Fig. 4 Sensitivity analysis for the model parameter $\eta$

We end this section with an analysis of the numerical convergence rate of the mixed finite element discretization (3.27), (3.28) of our model problem (3.22). Thereby, we shall investigate the sharpness of the error estimate (3.30). We choose the parametrization $r(u)=u^{1 / m}$ with $m \in \mathbb{N}, m>1$. Thus, problem (3.22) reads as

$-\Delta c+c^{1 / m}=f \quad$ in $\Omega=[0,1]^{2}, c=0 \quad$ on $\partial \Omega$. 
Fig. 5 Calculated collagen (top) and drug (bottom) profiles at time $T=240 \mathrm{~min}$ (left), $300 \mathrm{~min}$ (center) and $360 \mathrm{~min}$ (right) of a two-dimensional simulation (transversal cross section of a bacillary drug delivery system)
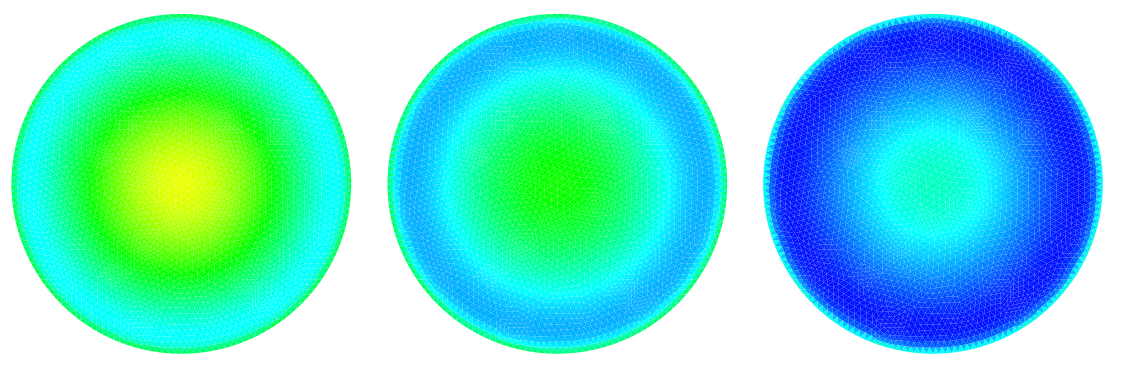

$1.00 \mathrm{E}+00$
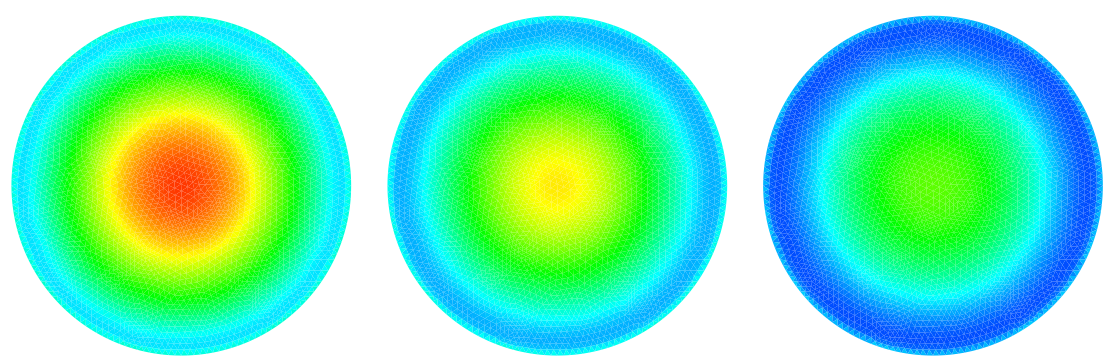

$1.36 \mathrm{E}+00$

1.17E+00

9.80E-01

7.90E-01

$6.00 \mathrm{E}-01$
Table 2 Test for convergence with Hölder-continuous nonlinearity $r\left(c_{\varepsilon}\right)=c_{\varepsilon}^{1 / m}$, $m=2$ and regularization parameter $\varepsilon=10^{-14}$

\begin{tabular}{lllll}
\hline$h$ & $\sqrt{\left\langle r\left(c_{\varepsilon}\right)-r\left(c_{\varepsilon, h}\right), c_{\varepsilon}-c_{\varepsilon, h}\right\rangle}$ & $\operatorname{Red}$ & $\left\|\boldsymbol{q}_{\varepsilon}-\boldsymbol{q}_{\varepsilon, h}\right\|_{\boldsymbol{L}^{2}(\Omega)}$ & $\operatorname{Red}$ \\
\hline $1.00 \mathrm{e}-1$ & $7.3601569 \mathrm{e}-03$ & - & $1.0793323 \mathrm{e}-02$ & - \\
$5.00 \mathrm{e}-2$ & $3.6884238 \mathrm{e}-03$ & 1.99 & $5.4532018 \mathrm{e}-03$ & 1.98 \\
$2.50 \mathrm{e}-2$ & $1.8451715 \mathrm{e}-03$ & 2.00 & $2.7387057 \mathrm{e}-03$ & 1.99 \\
$1.25 \mathrm{e}-2$ & $9.2268223 \mathrm{e}-04$ & 2.00 & $1.3716356 \mathrm{e}-03$ & 2.00 \\
$6.25 \mathrm{e}-3$ & $4.6134846 \mathrm{e}-04$ & 2.00 & $6.8621731 \mathrm{e}-04$ & 2.00 \\
\hline
\end{tabular}

\begin{tabular}{lllll}
\hline$h$ & $\sqrt{\left\langle r\left(c_{\varepsilon}\right)-r\left(c_{\varepsilon, h}\right), c_{\varepsilon}-c_{\varepsilon, h}\right\rangle}$ & Red & $\left\|\boldsymbol{q}_{\varepsilon}-\boldsymbol{q}_{\varepsilon, h}\right\|_{\boldsymbol{L}^{2}(\Omega)}$ & $\operatorname{Red}$ \\
\hline $1.00 \mathrm{e}-1$ & $1.1189781 \mathrm{e}-02$ & - & $1.1075110 \mathrm{e}-02$ & - \\
$5.00 \mathrm{e}-2$ & $5.6485600 \mathrm{e}-03$ & 1.98 & $5.4665501 \mathrm{e}-03$ & 2.02 \\
$2.50 \mathrm{e}-2$ & $2.8344437 \mathrm{e}-03$ & 1.99 & $2.7331187 \mathrm{e}-03$ & 2.00 \\
$1.25 \mathrm{e}-2$ & $1.4190860 \mathrm{e}-03$ & 2.00 & $1.3712282 \mathrm{e}-03$ & 1.99 \\
$6.25 \mathrm{e}-3$ & $7.0987767 \mathrm{e}-04$ & 2.00 & $6.9221081 \mathrm{e}-04$ & 1.98 \\
\hline
\end{tabular}

Table 3 Test for convergence with Hölder-continuous nonlinearity $r\left(c_{\varepsilon}\right)=c_{\varepsilon}^{1 / m}$, $m=4$ and regularization parameter $\varepsilon=10^{-14}$ $c(x, y)=x(1-x) y(1-y)$.

(3.27), (3.28) in a standard way; cf. [5]. We study the either cases $m=2$ and $m=4$. The calculated errors and error reduction factors, i.e., the ratios of the errors calculated for $h$ and $h / 2$ respectively, for a sequence of mesh sizes are summarized in Tables 2 and 3. In each mesh refinement step we reduce the mesh size $h$ by a factor of 2 .

In Tables 2 and 3 we observe an optimal first-order convergence behaviour of the mixed finite element discretization (3.27), (3.28) which would argue for the non-sharpness of our error estimate (3.30). However, we conjecture that the regularization (4.3) oversimplifies the test problem since $r^{\prime}\left(c_{\varepsilon}\right) \leq$ $r^{\prime}(\varepsilon)$, with $r^{\prime}(c)=c^{(1 / m)-1} / m$, is bounded from above whereas $r^{\prime}(c)$ becomes unbounded for vanishing values of $c$. Precisely, the regularization (4.3) makes the nonlinearity $r\left(c_{\varepsilon}\right)$ Lipschitz continuous, i.e., 
Table 4 Test for convergence with Hölder-continuous nonlinearity $r\left(c_{\varepsilon}\right)=c_{\varepsilon}^{1 / m}$, $m=2$ and regularization parameter $\varepsilon=h$

\begin{tabular}{lllll}
\hline$h$ & $\sqrt{\left\langle r\left(c_{\varepsilon}\right)-r\left(c_{\varepsilon, h}\right), c_{\varepsilon}-c_{\varepsilon, h}\right\rangle}$ & Red & $\left\|\boldsymbol{q}_{\varepsilon}-\boldsymbol{q}_{\varepsilon, h}\right\|_{\boldsymbol{L}^{2}(\Omega)}$ & $\operatorname{Red}$ \\
\hline $1.00 \mathrm{e}-1$ & $3.8458094 \mathrm{e}-03$ & - & $1.0814056 \mathrm{e}-02$ & - \\
$5.00 \mathrm{e}-2$ & $2.2116069 \mathrm{e}-03$ & 1.74 & $5.4661357 \mathrm{e}-03$ & 1.97 \\
$2.50 \mathrm{e}-2$ & $1.2477231 \mathrm{e}-03$ & 1.77 & $2.7420888 \mathrm{e}-03$ & 1.99 \\
$1.25 \mathrm{e}-2$ & $6.8900261 \mathrm{e}-04$ & 1.81 & $1.3723620 \mathrm{e}-03$ & 2.00 \\
\hline
\end{tabular}

\begin{tabular}{lllll}
\hline$h$ & $\sqrt{\left\langle r\left(c_{\varepsilon}\right)-r\left(c_{\varepsilon, h}\right), c_{\varepsilon}-c_{\varepsilon, h}\right\rangle}$ & Red & $\left\|\boldsymbol{q}_{\varepsilon}-\boldsymbol{q}_{\varepsilon, h}\right\|_{\boldsymbol{L}^{2}(\Omega)}$ & Red \\
\hline $1.00 \mathrm{e}-1$ & $3.5500634 \mathrm{e}-03$ & - & $1.0813337 \mathrm{e}-02$ & - \\
$5.00 \mathrm{e}-2$ & $2.1912031 \mathrm{e}-03$ & 1.62 & $5.4660022 \mathrm{e}-03$ & 1.98 \\
$2.50 \mathrm{e}-2$ & $1.3165121 \mathrm{e}-03$ & 1.66 & $2.7420423 \mathrm{e}-03$ & 1.99 \\
$1.25 \mathrm{e}-2$ & $7.6773342 \mathrm{e}-04$ & 1.71 & $1.3723438 \mathrm{e}-03$ & 2.00 \\
\hline
\end{tabular}

Table 5 Test for convergence with Hölder-continuous nonlinearity $r\left(c_{\varepsilon}\right)=c_{\varepsilon}^{1 / m}$, $m=4$ and regularization parameter $\varepsilon=h$ $\left|r\left(c_{\varepsilon}\right)-r\left(\tilde{c}_{\varepsilon}\right)\right| \leq L\left|c_{\varepsilon}-\tilde{c}_{\varepsilon}\right|$

for all $c_{\varepsilon}, \tilde{c}_{\varepsilon}$ satisfying $c_{\varepsilon}, \tilde{c}_{\varepsilon} \geq \varepsilon$ with Lipschitz constant $L=\varepsilon^{(1 / m)-1} / m$. In such a case, by a small modification of the proof of Theorem 2 the error bound

$$
\begin{aligned}
& \left\langle r\left(c_{\varepsilon}\right)-r\left(c_{\varepsilon, h}\right), c_{\varepsilon}-c_{\varepsilon, h}\right\rangle+\left\|\boldsymbol{q}_{\varepsilon}-\boldsymbol{q}_{\varepsilon, h}\right\|_{\boldsymbol{L}^{2}(\Omega)}^{2} \\
& \quad \leq C\left(h^{2}+\frac{1}{\varepsilon^{1-1 / m}} h^{2}\right)
\end{aligned}
$$

is obtained.

Due to the estimate (4.4), we conjecture that the convergence behavior of the considered mixed finite element approach is actually determined by the term $h^{2} / \varepsilon^{1-1 / m}$. To analyze this numerically, we repeat the previous convergence test, but now with choosing $\varepsilon=h$ instead of $\varepsilon=10^{-14}$ as done in Tables 2 and 3. Thus, for the square root of the lefthand side of (4.4) we may expect the order of convergence $3 / 4$ if $m=2$ and $5 / 8$ if $m=4$ and the error reduction factors $2^{3 / 4} \approx 1.68$ and $2^{5 / 8} \approx 1.54$, respectively. The calculated errors and reduction factors for a sequence of mesh sizes are summarized in Tables 4 and 5. In fact, the results of Tables 4 and 5, more precisely $\sqrt{\left\langle r\left(c_{\varepsilon}\right)-r\left(c_{\varepsilon, h}\right), c_{\varepsilon}-c_{\varepsilon, h}\right\rangle}$, argue for a non-optimal convergence behavior of the considered mixed finite element approach which however is slightly better than it can be expected from the error estimate (4.4) with $\varepsilon=h$. The reduction factors increase for $h \rightarrow 0$ which might be due to the fact that the function $c$ in (4.2) vanishes on the boundary of the unit square only and that the boundary layer or the singular behavior of $r^{\prime}(c)$ on $\partial \Omega$ is resolved better for $h \rightarrow 0$. Nevertheless, the calculated errors of Tables 4 and 5 clearly confirm a non-optimal convergence behavior of lowest-order Raviart-Thomas mixed finite element approximations of boundary value problems having reactive terms with unbounded derivative, as it is implied by Theorem 2 .

\section{Conclusions}

In this paper a mathematical model describing enzymatic degradation of collagenous drug delivery systems and simultaneous drug release was proposed. Existence and uniqueness of a solution of the model equations could be ensured. The mixed finite element approximation of the mathematical model was analyzed theoretically and numerically. In particular, a new error estimate of suboptimal order for reactive terms with unbounded derivative was proved. A sensitivity study was performed for the empirical parameters of the model equations and further parameters that are of importance for the application of the drug delivery systems in practice. The numerically calculated data were carefully compared with experimentally determined data. A good correlation between the either data was observed which shows the capability of the mathematical model to describe reliably enzymatic matrix degradation and simultaneous drug delivery.

Open Access This article is distributed under the terms of the Creative Commons Attribution Noncommercial License which permits any noncommercial use, distribution, and reproduction in any medium, provided the original author(s) and source are credited.

\section{References}

1. Adams, R.A.: Sobolev Spaces. Academic Press, New York (1975)

2. Bastian, P., Birken, K., Johanssen, K., Lang, S., Neuß, N., Rentz-Reichert, H., Wieners, C.: UG-A flexible toolbox for solving partial differential equations. Comput. Visualiz. Sci. 1, 2740 (1997)

3. Bause, M., Knabner, P.: Computation of variably saturated subsurface flow by adaptive mixed hybrid finite element methods. Adv. Water Res. 27, 565-581 (2004)

4. Bause, M., Merz, W.: Higher order regularity and approximation schemes for the monod model of biodegradation. Appl. Numer. Math. 55, 154-172 (2005) 
5. Brezzi, F., Fortin, M.: Mixed and Hybrid Finite Element Methods. Springer, New York (1991)

6. Chen, Z.: Expanded mixed finite element methods for quasilinear second-order ellipctic problems II. RAIRO Modél. Anal. Numer. 32, 501-520 (1998)

7. Cornish-Bowden, A.: Fundamentals of Enzyme Kinetics. Portland Press, London (2004)

8. Friess, W.: Drug Delivery Systems Based on Collagen. Shaker, Aachen (2000)

9. Fujita, H.: Diffusion in polymer-diluent systems. Fortschr. Hochpolym. Forsch. 3, 1-47 (1961)

10. Huang, A.A.: Kinetic studies on insoluble cellulose-cellulase system. Biotechnol. Bioeng. 17, 1421-1433 (1975)

11. Ladyženskaya, O.A., Solonnikov, V.A., Ural'ceva, N.N.: Linear and Quasilinear Equations of Parabolic Type. American Mathematical Society, Providence (1968)

12. McLaren, A.D.: Enzyme reactions in structurally restricted systems, IV. The digestion of insoluble substrates by hydrolytic enzymes. Enzymologia 26, 237-246 (1963)

13. Merz, W.: Strong solutions for reaction-drift-diffusion problems in semiconductor devices. ZAMM 81, 623-635 (2001)

14. Merz, W.: Global existence result of the monod model. Adv. Math. Sci. Appl. 15, 709-726 (2005)

15. Metzmacher, I.: Enzymatic Degradation and Drug Release Behavior of Dense Collagen Implants. Ph.D. Thesis, LMU University of Munich, Dr. Hut Verlag, München (2005)

16. Metzmacher, I., Radu, F.A., Bause, M., Knabner, P., Friess, W.: A model describing the effect of enzymatic degradation on drug release from collagen minirods, Eur. J. Pharm. Biopharm. 67(2), 349-360 (2007). doi:10.1016/j.ejpb.2007.02.013
17. Quarteroni, A., Valli, A.: Numerical Approximation of Partial Differential Equations. Springer, Berlin (1997)

18. Radu, F.A., Bause, M., Knabner, P., Friess, W., Lee, G.: Modelling of drug release from collagen matrices. J. Pharm. Sci. 91, 964972 (2002)

19. Radu, F.A.: Mixed finite element discretization of Richards' equation: error analysis and application to realistic infiltration problems. Ph.D. Thesis, University of Erlangen-Nürnberg (2004)

20. Radu, F.A., Pop, I.S., Knabner, P.: Order of convergence estimates for an Euler implicit, mixed finite element discretization of Richards' equation. SIAM J. Numer. Anal. 42, 1452-1478 (2004)

21. Rubingh, D.N., Bauer, M.D.: Catalysis of hydrolysis by proteases at the protein-solution interface. Stud. Polym. Sci. 11, 445-464 (1992)

22. Thombre, A.G., Himmelstein, K.J.: A simultaneous transportreaction model for controlled drug delivery from catalyzed bioerodible polymer matrices. AIChE J. 31, 759-766 (1985)

23. Tzafriri, A.R.: Mathematical modeling of diffusion-mediated release from bulk degrading matrices. J. Contr. Release 63, 6979 (2000)

24. Tzafriri, A.R., Bercovier, M., Parnas, H.: Reaction diffusion model of the enzymatic erosion of insoluble fibrillar matrices. Biophs. J. 83, 776-793 (2002) 